\title{
Las TIC como desarrollo personal y social
}

\section{The TICS as personal and social development}

\author{
Daniela Salazar Ramírez' \\ Universidad Nacional Abierta y a Distancia
}

\begin{abstract}
Resumen
En este artículo se encontrarán los diferentes contrastes que a través del tiempo las tecnologías de información y comunicación han generado en la educación y desarrollo del individuo, creando una perspectiva sobre la evolución de la sociedad y como contribuyen en su crecimiento. La educación cada vez más va introduciendo métodos con los cuales los estudiantes se sientan identificados y satisfechos en su realización, como lo es la cultura virtual en su totalidad con sus tecnologías.
\end{abstract}

Palabras clave: educación, evolución, desarrollo, sociedad, tecnología.

\begin{abstract}
In this article it will find the different contrasts that over time the information and communication technologies have generated in the education and development of the individual, creating a perspective on the evolution of society and how it contribute to their growth. Education increasingly has introduced methods with which students are identified and satisfied in their fulfillment, as it is in the virtual culture as a whole with its technologies.
\end{abstract}

Keywords: education, evolution, development, society, technology.

Recibido: 24 de octubre de 2015

Aceptado: 15 de noviembre de 2015

Las tecnologías de la información y comunicación han generado un gran avance en todos los campos de la educación, de la ciencia y la economía, este nuevo acceso a la información le muestra a las personas que tienen la posibilidad de conocer y aprender sobre lo que sea, cuando sea. Para esto, se hizo indispensable llevar el conocimiento de estas tecnologías a todos los rincones del país, incluyendo a las comunidades vulnerables y socialmente aisladas, reconociendo que la llegada de las TIC ha revolucionado innegablemente al mundo.

Con el tiempo se deben ir creando mejores alternativas de acceso y no se puede dejar que el individuo no se sienta identificado con su sociedad, se debe sentir parte de una sociedad que los vincula y no excluye por sus costumbres, condición económica, tradiciones, opción sexual, o pensamiento; ya que si hablamos de un desarrollo social, dependemos también de la forma en que ha influido la globalización en cada comunidad, donde habrá ventajas y desventajas, pero no podemos retroceder y cubrir los defectos que tenemos como seres humanos, al contrario empezando por la aceptación y más alternativas donde el voto de la persona tenga el valor que debe ser.

Se debe tener en cuenta que se estará expuesto a estos cambios, y se debe saber adaptar en su medida, reconociendo aquello que puede ayudar a un desarrollo tanto personal como social.

1 Estudiante de Psicología, Universidad Nacional Abierta y a Distancia, CEAD Palmira, Colombia. Correo: danisalaz1994@hotmail.com 
En la medida en que el ser humano avanza también ira evolucionando la sociedad, como conjunto integrado por individuos adaptables y cambiantes, buscando siempre la unión de conocimientos a través de un solo medio, como lo es la tecnología, que provee la posibilidad de visualizar tradiciones y costumbres ancestrales, comparándolas con tendencias que se viven en la actualidad, dándo como resultado una raza consciente de su existencia y de lo que debe atravesar y averiguar para una realización global.

Siendo las TIC un conjunto de nuevas herramientas que ha creado el hombre en busca de su necesidad de satisfacer insuficiencias de comunicación, permiten de este modo generar ambientes en los que, a pesar de las distancias, no existan barreras para mantener una constante comunicación con el conocimiento y las personas; es en este punto donde se ve reflejado el comportamiento de una sociedad que se adapta fácilmente a los cambios tecnológicos creados por el mismo ser humano para su desarrollo.

Los medios de comunicación (desde la imprenta, la radio, la televisión y ahora Internet) se han convertido en arenas, ámbitos y actores fundamentales en la transformación de la vida pública de sociedades modernas. (Bonilla, 2005, pág. 59)

A través de la historia todos estos medios de comunicación, creados por el mismo hombre, han ido siendo indispensables para la vida humana y se puede decir que en un futuro el desarrollo que se haya tenido será significativo. A pesar de que aún falta cobertura, se hace lo posible para que todos puedan tener acceso a las TIC, como una herramienta educativa donde gracias a estas tecnologías, que utilizadas a trav\{es de la internet, se logren tener a mano formas prácticas de interactuar, compartir, enseñar, aprender y estudiar, cambiando las perspectivas pedagógicas de distancia y tiempo.

La educación a través de la mediación virtual se ha ido incluyendo aún más en la sociedad; por los beneficios y ventajas en el autoaprendizaje y la autonomía que los individuos adquieren en sus aulas, generando así una apropiación de su rol, igualmente de la metodología aplicada para la diversidad sociocultural y para comunidades e individuos con poco o limitado acceso a la educación y, principalmente, una identificación con su pedagogía.

Se busca que los estudiantes logren una excelente adaptación al modelo educativo, dando respuestas a sus inquietudes y acompañamiento en sus labores creando herramientas y estrategias que puedan optimizar su rendimiento, logrando así que tengan responsabilidad, permanencia, cultura virtual y un perfil que lo desempeñen como un sujeto dispuesto a desarrollarse en distintos tipos de áreas.

La autora se encuentra formandose como Psicóloga en la Universidad Nacional Abierta y A Distancia - UNAD, cursando el VI semestre, con habilidades educativas que por medio de la pedagogía virtual ha adquirido, considerándose alguienquequiere constantementealimentarsus conocimientos y así tener bases fundamentales para desarrollarse en la sociedad.

El interés por seguir formándose en esta institución, radica en transmitir sus saberes y experiencias propias a los estudiantes que en los primeros semestres se sientan inestables en su vocación, generando diálogos y estrategias para que no deserten, esclareciendo las oportunidades y ventajas que existen en esta metodología.

Como estudiantes se necesita mantener un impulso por adquirir más conocimientos sobre las labores que realiza la universidad en sus áreas, y poner aún más en práctica aquellos adquiridos en el transcurso de los años, tanto a nivel intelectual como tecnológico.

Contribuir de manera significativa a la UNAD requiere equilibrar el aprendizaje de los estudiantes a través de su formación $y$, así mismo, continuar fortaleciendo la calidad con nuevas estrategias, resaltando el uso de las TIC. La importancia del rol que cumplen las TIC, surge de la necesidad de la interacción directa entre el estudiante y la universidad, sin apreciar la distancia como un impedimento para la comunicación. 
La experiencia que se adquiere mediante la vinculación a este tipo de tecnologías, se basa fundamentalmente en el manejo y conocimiento de las culturas virtuales, creciendo en nuevas y mejores habilidades para un eficiente $y$ eficaz autoaprendizaje, creando autonomía y mostrando a la sociedad una evolución que aprovecha al máximo los recursos para superar barreras.

\section{Referencias}

Berger, P. L. (1986). La construcción Social de la Realidad. H. F. Martínez de Murgia. Argentina: Paidos Ibérica.

Bonilla, J. I. (2005). Apuntes sobre Medios de Comunicación, esfera pública y democracia. En Comunicación para construir público. Bogotá: Convenio Andrés Bello. 